\title{
Interactive Visualisation of MRI Vector and Tensor Fields
}

\author{
Abhir Bhalerao $^{1}$ and Paul Summers ${ }^{2}$ \\ 1 Department of Computer Science \\ University of Warwick, UK \\ abhir@dcs.warwick.ac.uk \\ 2 Clinical Neurosciences \\ Kings College Medical School, UK \\ p.summers@iop.kcl.ac.uk
}

Methods for visualising and analysing MR phase images that depict motional properties of blood or molecular diffusion in a way that meets the needs of the expert users has been a topic of intense study in recent years e.g., [2, 3]. The clinical attraction of phase contrast imaging has been its ability to depict flowing blood whereas diffusion imaging is associated both with delineating strokes and identifying patterns or pathways of connectivity within the brain. There are similarities in the pre and post-operative questions asked by experts for these two types of MR images, e.g. does a particular vessel feed or drain a given region or what are the terminal connections of a neuronal tract, and what is the flow pattern in a given region or what is the connectivity of a cortical region.

We have developed a graphical tool, Angiotool [1], that attempts to meet some of the requirements of clinical experts by providing a set of low-level and semi-automated visualisation and analysis tools - low-level in this context meaning they are accountable and their effects transparent to the user. The tools are also consistent in the data navigation and interaction interfaces, to some extent, across MR image types.

The orthogonal views can show both slice data and MIPs (Figure1). Pointand-click cursor navigation in the slice data continues to operate on the MIP image allowing any image feature, such as a vessel, to be manually tracked in the other two views which are automatically centred on the cursor position. The render window maintains a depth buffer of the current projection from which the cursor position can readily be mapped. Also, a limited MIP, where image voxels between a pair of depth planes either side of the cursor position, can be used to prevent depth ambiguity errors which may result from the projection of brightest pixels. The $3 \mathrm{D}$ analysis window is linked to the the render window and displays results of analyses on the volume data. An examples of such an analyses iis tracking, either as flow stream generation or as the derivation of neuronal tract orientation. Flow tracking experiments involve setting one or more particles (or seeds) into the velocity field (Figure1(a)-(c),(e)). The tracking process is local and relies solely on the velocity data at each point using a physical space, point tracking algorithm. Tracking in diffusion data is performed on a derived vector field such as the principal eigenvector which represents the 
local anisotropy modulated by a scalar or the fractional anisotropy index which measures the eccentricity of the tensor. Simulation results, such as flow streams, are buffered by Angiotool which can be recorded or replayed.

(a)

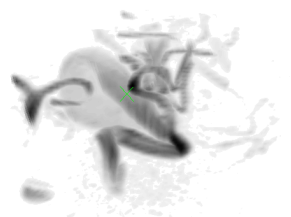

(d)

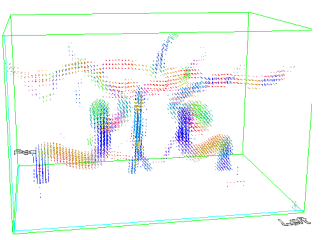

(e)

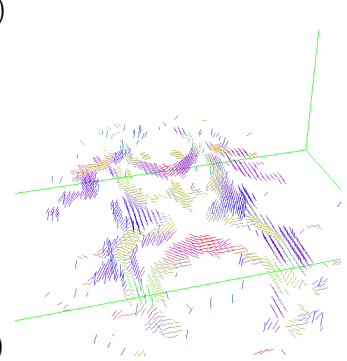

(b)

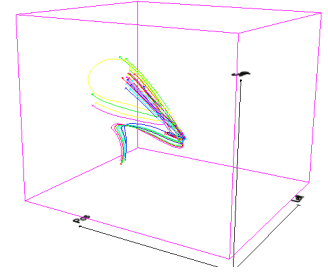

(c)

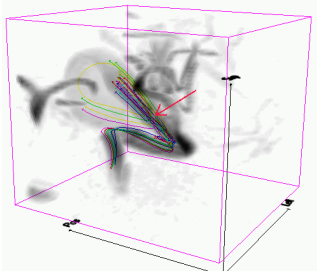

(f)

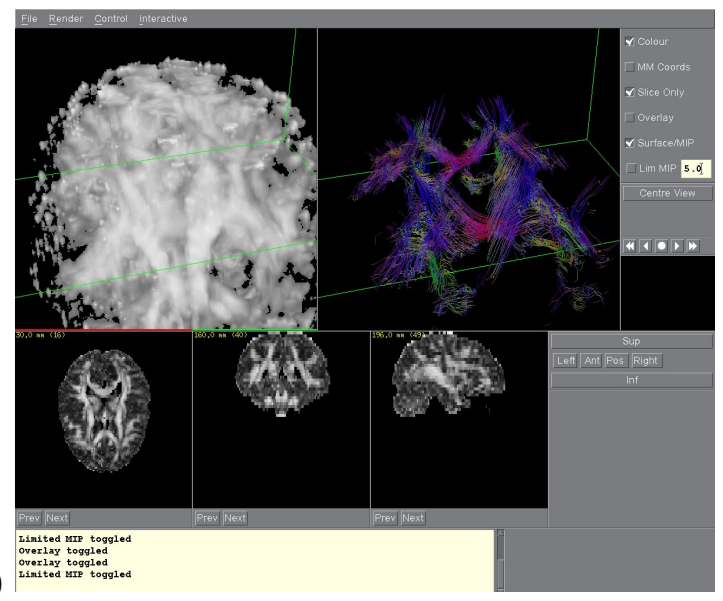

Fig. 1. Flow tracking experiments to determine the neck of a giant cerebral aneurysm: (a) User selects starting point(s) in MIP view. (b) By reversing the direction of flow from the seed point, blood flow is followed from within the aneurysm back through the neck and into the feeding artery. (c) Overlay of MIP with flow experiment precisely locates the neck. (d) Example of velocity data depicted as a field of vectors. (e) Plot of principal eigenvectors across a slice from an example diffusion image. (f) Overview of diffusion tensor image analysis: all tracks from a central slice across the brain. The MIP and orthogonal views show a scalar (derived) image of fractional-anisotropy using a magnitude image for the tensor tracking.

The current implementation is built upon open standards technologies: GUI toolkits using $\mathrm{X}$ and Motif and 3D graphics using OpenGL. While not as extensible or general purpose as comparative surgical planning and analysis tools, we believe that the presented GUI model could usefully form the basis of other clinical applications of this type. 


\section{References}

[1] A. Bhalerao and P. Summers. Angiotool: A Tool for Interactive Visualization of MRI Vector and Tensor Fields. Research Report CS-RR-382, Department of Computer Science, University of Warwick, Coventry, UK, May 2001.

[2] T. M. Koller. From Data to Information: Segmentation, Description and Analysis of the Cerebral Vascularity. PhD thesis, Swiss Federal Instititute of Technology, Zurich, 1995.

[3] C-F. Westin, S. E. Maier, B. Khidhir, P. Everet, and F. A. Jolesz nd R. Kikinis. Image Processing for Diffusion Tensor Magnetic Resonance Imaging. In Proc. of MICCAI'99, pages 441-452, 1999. 